\title{
CrimRxiv
}

\section{Whether \& How To Share a Single Work Across Multiple Platforms}

Published on: Apr 08, 2021

DOI: $10.21428 / \mathrm{cb} 6 a b 371 . c 51 e 1 c 78$

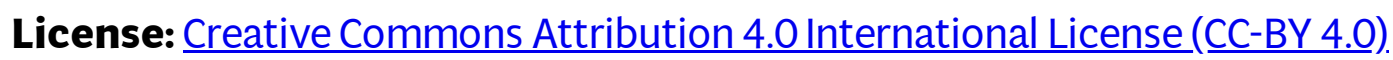


There are many platforms, or places, to make your papers green open access (OA). In addition to CrimRxiv, there is your personal webpage, institutional repository, other field-specific repositories, broader repositories, and websites like ResearchGate and Academia. Should you use all of them? Or which ones? And how so? This page answers those questions.

\section{What Should Platforms Do For You?}

We should begin by outlining how platforms spread your scholarship and increase your impact; namely, by making your work findable, browsable, dynamic, and legal to immediately share. You may be more interested in one of those than the others, but all of them are important-increasingly so.

\section{Findability Is the Minimum Goal}

At a minimum, you want a platform to make your works findable on Google and other search engines. That way, if a potential reader knows of your paper and wants to find a free version, they can do so by searching the web. All of the aforementioned places automatically enable that, some better than others (e.g., higher rank in search results). Better yet is to be indexed in Google Scholar, as the search results are accompanied by more information. For example, the site shows how many times, and by whom, a paper was cited. Not all websites are indexed by the service. The inclusion criteria are on this page. The standards are less often met by personal sites. They also tend to have inferior metadata to other platforms and lack the ability to easily assign DOIs. Those problems limit the impact of personal sites, as measured by page views and unique users (per different IP addresses). This means that, for most authors, their personal websites should not be a priority platform. $\underline{1}$

\section{Browsability Is a Higher Goal}

In addition to being findable, you want your papers to be browsable: seen by others who are looking for work on a topic. Even if a potential reader does not know of your paper, they can find it by scrolling through a webpage. Discoverability, you could say, equals findability plus browsability. Personal websites are worthless for browsing. On ResearchGate and Academia, it is not possible to browse per se, as everything is search-based. $\underline{2}$ It will not be rational, usually, for someone to browse criminology on any given institutional repository or another big repository, like SocArXiv, SSRN, or Zenodo. $\underline{3}$ You can browse those sites by “Subject," “Network \& Subject Area," or 
"Community," respectively, but the results are too narrow, sparse, or incomplete to be usefully browsed. The best place for browsing a specific field is a field-specific repository. CrimRxiv is criminology's repository.

\section{Embedding Is a Higher Goal Still}

In the future, there will be two classes of platforms: those that enable embedding dynamic materials into papers, and those that do not. Articles, for example, will include interactive figures, videos, and audio clips. They will also include the very data and code used to create the findings. To be clear, we are not talking about hyperlinking out to such things, but rather including them within the body of a paper. This will not only transform how scholarship is disseminated, but also how research is done.

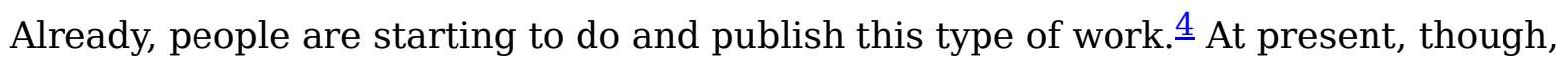
no repository enables embedding dynamic content-with the exception of those on $\underline{\text { PubPub}}$, as is CrimRxiv.

\section{Legality Is Essential}

Copyright agreements impede the free sharing of criminological papers. Not only do they allow paywalling versions of record, but also embargoing green access to postprints. However, other agreements can preempt and thereby effectively nullify exclusive rights with publishers. We are referring to Harvard-style rights-retention licenses in which authors give another entity a nonexclusive, waivable right to share their future works. Until recently, only funders and academic institutions have rightsretention policies, so only their authors are protected by them. This is an important problem that we solved by offering an Individual Open Access License. By signing the license, you make it legal for CrimRxiv to distribute your future papers (when you please), irrespective of publisher embargoes. If you are not covered by a Harvard-style license, you should sign ours. We are the only platform that offers one.

\section{Whether \& How To Share a Single Work Across Multiple Sites}

With that background, we return to the questions: Should you use all of the platforms ? Which ones? And how so?

\section{Which Platform(s) Should I Use?}

The answer to the first question is "No, that's impractical." It also is irrational. Strictly with respect to findability, there is no benefit to using more than one platform so long as its contents are sufficiently findable. Actually, there is a potential penalty: some search engines, like Google, can lower your contents' rank if there are too many copies 
of it. All of the aforementioned platform options are findable. Perhaps the better question, then, is "Which platform(s) should you use?" If you want your papers to be findable then any platform is ok, though personal websites likely will be the worst. If you want your papers to be browsed along with other criminology papers, then the best option is CrimRxiv. $\underline{5}$ If you want the ability to embed dynamic materials within your papers, then the only option is CrimRxiv. Ditto if you want to legally preempt embargos by signing a rights-retention license. $\underline{6}$ Thus, by using CrimRxiv, you will increase your impact in ways that you cannot with other platforms. $\underline{7}$

\section{Which Platform Should Be Used First?}

If you are set on using multiple platforms to share a work, $\underline{8}$ you should think through which site to publish it on first. That choice dictates which page serves as the "original" versus a "copy." You should make it easy for software and people to determine which is which. Preferably, those linkages and designations are in the metadata (e.g., PubPub uses the "Connections"). Short of that, each copy of a paper should have a clear statement that points to the original. For example, the statement may simply read: "This paper is a copy of the version published at 'this DOI'." There is no one right way to decide where to put the original. We think it is logical to prioritize the place that offers the most utility, such as in findability, browsability, embedding possibilities, and by providing an Individual Open Access License. The first two and the last one increase access to your work. The third makes it more interesting.

\section{Illustrative Video For How to Link a Paper Across Platforms and Designate a Copy as Such}




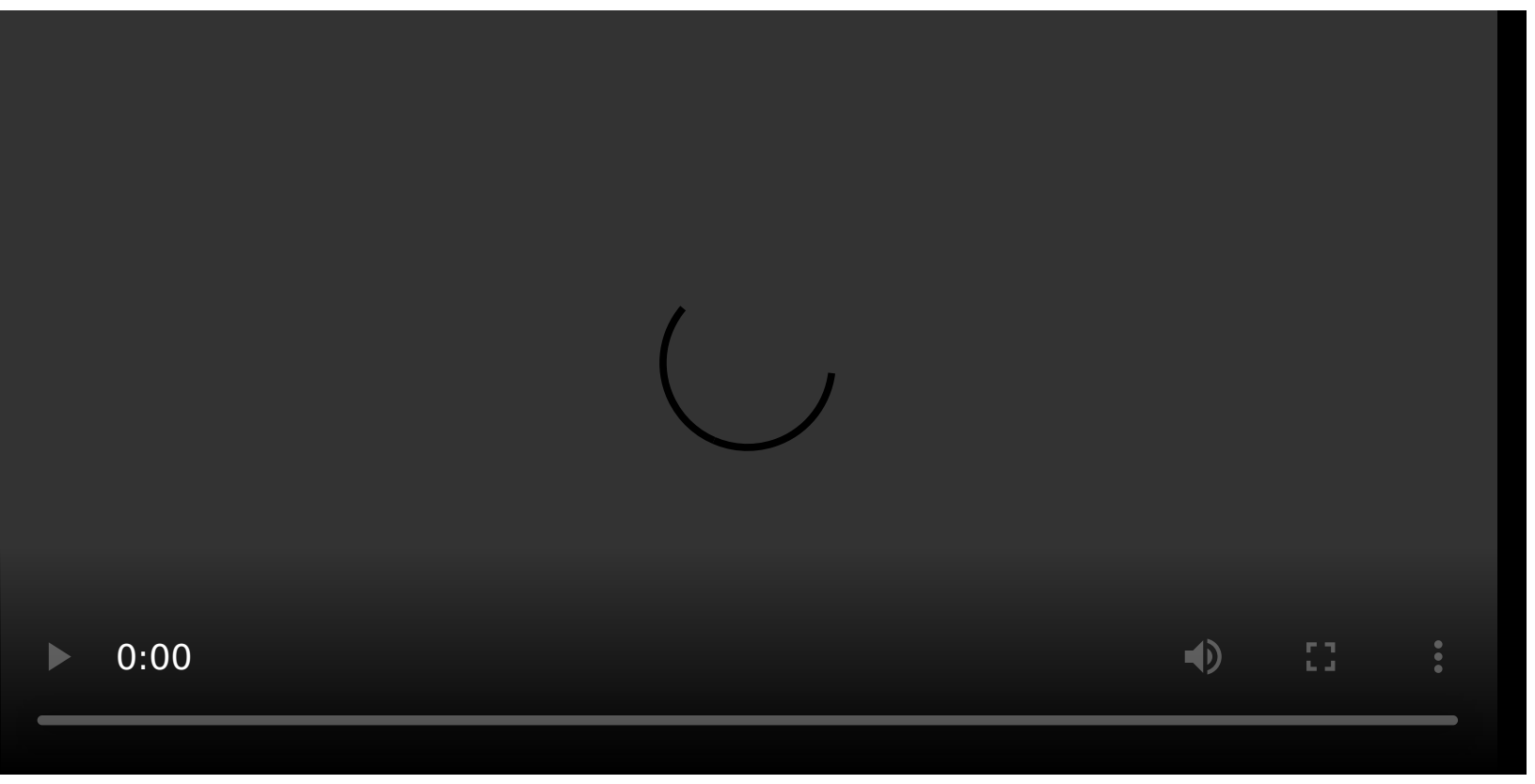

Illustrative Video For How to Use the "Connections” Feature of CrimRxiv

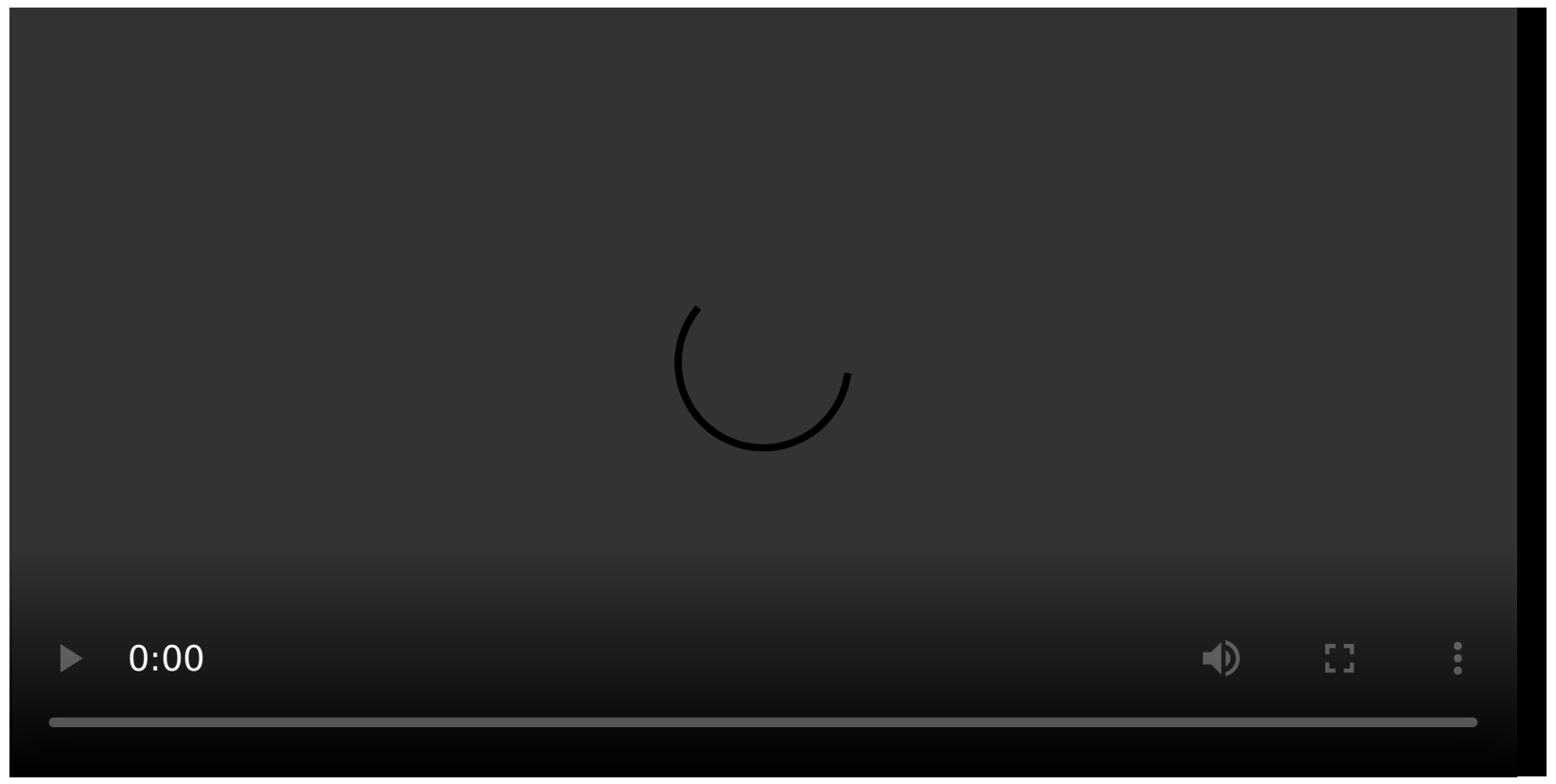

\section{Footnotes}

1. However, there is a free, easy, and highly functional answer to those problems:

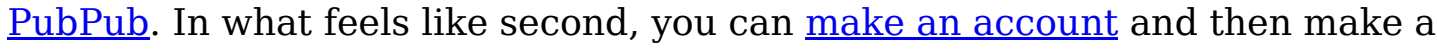
"Community" in your name. By doing your personal webpage that way, you ensure it is findable, with equal or better data than other share points, with the ability to 
assign DOIs. It cannot be overemphasized: Assigning DOIs is essential to a stable, long-living record of digital objects. Not only that they continue to exist, but also that their properties are known (e.g., which came first and provenance). $\subseteq$

2. We could be wrong about their browsability. $\_$

3. A user of SocArXiv could $\subseteq$

4. See, for example, ...Qualitative...Criminology..

5. This statement assumes sufficient uptake in due time. $\_$

6. Unless, that is, you are already covered by a funder's or academic institution's rights-retention policy. $\triangleq$

7. Whether and why to use any given platform is a judgment call, which may be based on reasons other than findability, browsability, and embedding capability. Common sense suggests that a personal website serves a marketing function. ResearchGate and Academia have the same allure. It is conscionable to share your work in the repository of the institution that pays your salary. You may prefer SocArXiv, SSRN, or Zenodo because they are popular or for another reason. $\_$

8. Any given paper can have multiple versions (e.g., preprint, postprint), each with their own original (as should be indicated by a DOI), and, possibly, a "copy" or "copies" (which may have their own DOIs). $\_$ 